\title{
EVALUATION OF PATIENTS UNDERGOING TRANSARTERIAL CHEMOEMBOLIZATION (TACE) FOR HEPATOCELLULAR CARCINOMA WITH RESPECT TO TUMOR SIZE, SITE AND NUMBER OF LESIONS.
}

\author{
Amjad Sattar ${ }^{1}$, Shabbir Naeem ${ }^{2}$, Ahsan Ali' \& Kiran Nazeer ${ }^{2}$ \\ Dow University of Health Sciences. \\ Sindh Institute of Urology and Transplant.(SIUT).
}

Corresponding author: amj7777@hotmail.com

\begin{abstract}
TACE is the combination of two procedures, an infusion of the mixture of chemotherapeutic agents (e.g. doxorubicin, cysplatin, or mytomycin C) and hepatic artery embolization in which particles such as polyvinyl alcohol or gelfoam, are injected simultaneously into selected branches of the hepatic artery supplying the tumor. Hepatocellular carcinoma is rarely seen before the age of 40 years and reaches a peak at around 70 years of age. Owing to its multicentricity, large tumor size and pre-existing cirrhosis most of these tumors were deemed unrespectable at the time of diagnosis. The purpose of this study was to evaluate the tumor size, site and number of lesions that is acquired on hepatic angiogram during TACE of the liver, and compare it with the information provided by pre-interventional contrast enhanced CT images as there is a possibility that CT may not show smaller lesions. This study was carried out in the Department of Angiography, Sindh Institute of Urology and Transplantation (SIUT) from May to October 2011. A total of 30 patients was included in the study. All individuals underwent pre-procedural imaging with contrast material, enhanced CT angiography or MR to ensure that the disease was liver-dominant and to visualize the blood vessels of the liver. This study included 30 patients age ranges 40-80 years, 22 of whom were males and 8 of them were females. HCC had been diagnosed in all patients. Each patient underwent selective Hepatic Chemoembolization in either the right, left or in both lobes. . In comparison to CT, the hepatic angiogram showed 14(46.6\%)patients having single lesion, $7(23.3 \%)$ were having double lesions and $9(30 \%)$ were having multiple lesions. So the overall sensitivity of hepatic angiogram in the detecting number of lesions is $75 \%-80 \%$. The size of $\leq 2 \mathrm{~cm}$ HCC was found in $12(35 \%)$ patients, HCC $>3 \mathrm{~cm}$ were found in $7(21 \%)$ patients and $\mathrm{HCC}>4 \mathrm{~cm}$ was found in 15(44.4\%) patients. The size of the HCC which we found in most of the patients during our study is $>4 \mathrm{~cm}$.
\end{abstract}

\section{KEYWORDS}

TACE, hepatic artery embolization, Hepatocellular carcinoma, Hepatic Chemoembolization

\section{INTRODUCTION}

Liver cancer is the fifth most common cancer in the world among the male sex, taking the second place with respect to cancer related deaths. Conversely in the females it is the seventh most common cancer diagnosed and sixth leading cause of death(Wang Y, 2013). In Pakistan, the most common etiological factors have been reported as hepatitis B and aflatoxins(Kausar S 1998). The most common form of primary liver cancer is the hepatocellular carcinoma. Hepatocellular carcinoma is rarely seen before the age of 40 years and reaches a peak at around 70 years of age. Owing to its multicentricity, large tumor size and preexisting cirrhosis most of these tumors were deemed unresectable at the time of diagnosis(Forner A, 2012). Due to these facts the transarterial chemoembolization (TACE) has gained immense popularity in the management of this disease(Llovet JM 2008).

The basis of TACE is the fact that the majorityof the blood supply of hepatocellular carcinoma is derived from the hepatic artery. Therefore TACE is used either as an adjuvant therapy or as a palliative procedure for the unresectable disease. It has been shown that TACE prior to surgical resection can not only prevent recurrence of carcinoma but also facilitate surgery by reducing tumor bulk $\underline{\mathrm{Xu} \mathrm{KC}}$ 2009).

TACE is the combination of two procedures, an infusion of the mixture of chemotherapeutic agents (e.g. doxorubicin, cysplatin, or mytomycin C) and hepatic artery embolization in which particles such as polyvinyl alcohol or gelfoam, are injected simultaneously into selected branches of the hepatic artery supplying the tumor. Since the hepatic artery supplies nearly $100 \%$ of the blood supply of the malignant tumours of the liver, this renders the tumor tissue ischemic and hence accounting for the reduction in tumor size. Also this fact allows for higher intracellular concentrations of chemotherapeutic agents resulting in tumor necrosis while limiting damage to non timorous liver(Transarterialchemoembolization Accessed on June 2013).

The purpose of this study was to evaluate the tumor size, site and number of lesions that is acquired on hepatic angiogram during TACE of the liver, and compare it with the information provided bypre-interventionalcontrast enhanced $\mathrm{CT}$ images as there is a possibility that CT may not show smaller lesions.

\section{MATERIALS AND METHOD}

This study was carried out in the Department of Angiography, Sindh Institute of Urology and Transplantation (SIUT) from May to October 2011. A total of 30 patients was included in the study. Informed consent was taken from all the patients. These patients were referred from the Gastroenterology Department for TACE after being diagnosed as hepatocellular carcinoma.

TACE was performed in angiography unit. All procedures were performed on the Digitalsubstraction angiography machine (Toshiba $\mathrm{KXO100} \mathrm{G)} \mathrm{at} \mathrm{frame} \mathrm{rate} 3 \mathrm{fr} / \mathrm{s}, 76 \mathrm{kvp}, 160 \mathrm{~mA}$ and $39 \mathrm{~ms}$ as per Department protocol. Medical, Surgical and contrast related history was taken and Informed consent was signed from all patients undergoing TACE.

All individuals underwent pre-procedural imaging with contrast material, enhanced CT angiography or MR to ensure that the disease was liver-dominant and to visualize the blood vessels of the liver. The main portal vein was ensured to be patented adequate portal perfusion was confirmed and collateral flow was ensured with hepatoportal flow (i.e. blood flowing toward the liver).

Pre-procedural patient preparation included hydration, fasting, steroids, bowel preparations and laboratory evaluation including complete blood count, prothrombin time, bleeding time,clotting time (INR), creatinine, urea and evaluation of liver and kidney function. Subsequently, after all aseptic measures a local anesthetic was applied and a puncture was made in the common femoral artery with the help 
CAdvance Educational Institute \& Research Centre - 2013 www.aeirc-edu.com

of $19 \mathrm{G}$ Angio needle. Standard guide wire $0.035 \times 150 \mathrm{~cm}$ was introduced into the needle; 5fr angiographic vascular sheath was slided over the guide wire after the removal of the needle. A selective angiogram of the celiac trunk and superior mesenteric artery was performed to determine the origin and course of tumor-feeding vessels. Using micro catheter the feeding artery of HCC was cannulated super selectively and chemoembolic material and lipoidal injected. Pre medications include intravenous injection of $3 \mathrm{cc}$ nalbuphine ( $1 \mathrm{ml}$ diluted in $9 \mathrm{cc}$ of saline) and metoclopramide. Post procedure angiogram was done and repeated spot films were taken to confirm that the tumor had been optimally treated.

The post procedure care included management of the puncture site regarding bleeding and hematoma. Vitals were taken after every 30 minutes for 4 hours.Patients were asked not to move for 6hours at least. The post procedure medication for pain was administered to all patients.

\section{RESULTS}

This study included 30 patients age ranges $40-80$ years, 22 of whom were males and 8 of them were females. HCC had been diagnosed in all patients. Each patient underwent selective Hepatic Chemoembolization in either the right, left or in both lobes. All procedures were performed successfully, without immediate (within 24 hours after chemoembolization) complications.

InCT Triphasic study out of 30 patients, we found $17(56.6 \%)$ patients having single lesion, 6 (20\%) were having double lesions, 6 (20\%) were having multiple lesions and 1(3.3\%)of the patient having no lesion. In comparison to CT, the hepatic angiogram showed 14(46.6\%) patients having single lesion, 7(23.3\%) were having double lesions and 9 (30\%) were having multiple lesions. So the overall sensitivity of hepatic angiogram in detecting number of lesions is $75 \%-80 \%$. (Figure 01)

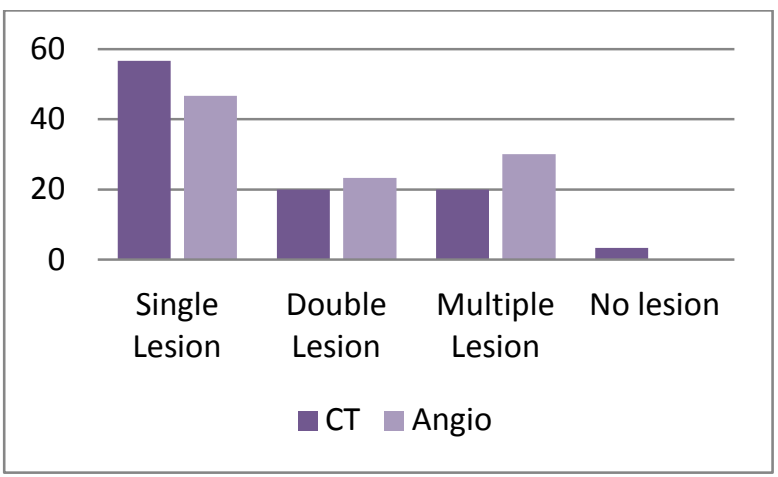

Figure 01: Number of lesions

Further on pre procedural imaging we found 5(16.6\%)patients having alesion in Left Lobe of Liver, 21(70\%) patients having lesion in Right Lobe and $4(13.3 \%)$ patients were having lesion occupying both Lobes. Its mean that Right lobe of the Liver is more sensitive in having HCC lesion. (Table 01)

\begin{tabular}{|l|l|}
\hline Site of Lesion & No of Patients \\
\hline Lesion in Left Lobe & $5(16.6 \%)$ \\
\hline Lesion in Right Lobe & $21(70 \%)$ \\
\hline Lesion in Both Lobes & \\
\hline
\end{tabular}

Table 01: Site of Lesions
The size of $\leq 2 \mathrm{~cm}$ HCC were found in $12(35 \%)$ patients, $\mathrm{HCC}>3 \mathrm{~cm}$ were found in 7(21\%)patients and $\mathrm{HCC}>4 \mathrm{~cm}$ was found in 15(44.4\%)patients. The size of the HCC which we found in most of the patients during our study is $>4 \mathrm{~cm}$. (Figure 02)

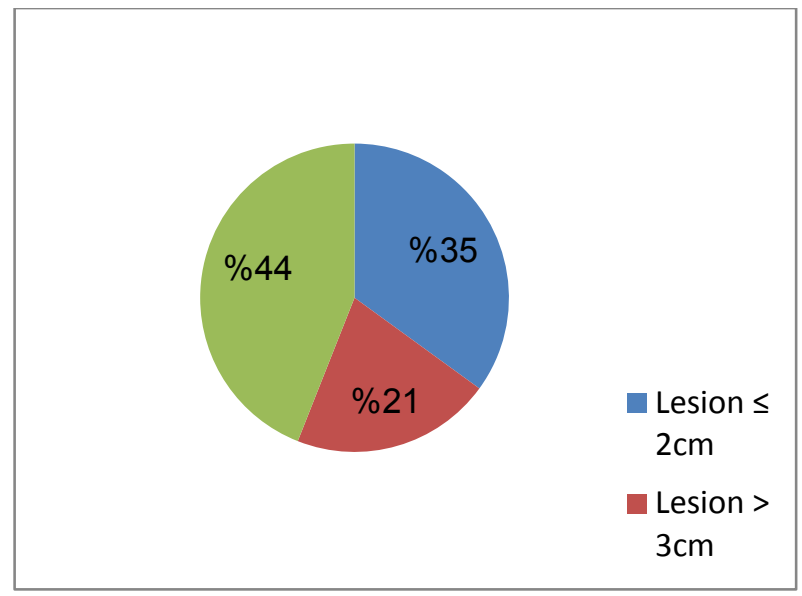

Figure 02: Size of Lesion

\section{DISCUSSION}

Hepatic vascular interventions such as TACE are most likely to succeed when the target lesions are accurately identified $b$ real-time angiography. However, owing to insufficient tumor vascularity or irregular enhancement of the background cirrhotic parenchyma, only $77 \%$ of hepatic tumors identified with previous MDCT are visualized with digital subtraction angiography(Iwazawa J 2010).

In the preoperativeevaluation of hepatic tumors, the imaging studies used must be highly sensitive and specific for the detection of malignant neoplasms to enable the selection of appropriate surgical candidates and avoid unnecessary surgery. For this several imaging techniques are used to detect HCC. Several authors have evaluated the accuracy of CT imaging in detecting HCC nodules. They used histologic assessment of liver biopsy specimens, liver parenchyma taken by partial liver resection, or explanted liver at the time of liver transplantation as references and have reported a wide variety of sensitivities ( $40 \%$ to $>90 \%$ ). Still, the question of whether Hepatic angiogram provides information beyond that obtained with other noninvasive modalities, as preoperative evaluation in the era of MDCT, remains to be addressed.

In recent years, a few studies have evaluated the role of multiphasic scanning by MDCT in detecting small hypervascular HCC. They have drawn a conclusion that the utility of faster speed and thinner slice $(6 \mathrm{~mm})$ MDCT can improve the detectability of hypervascular small HCC, and additional early arterial phase imaging does not improve the detection of HCC compared with Tiphasic CT images (Zheng XH, 2005).With this in mind, we reviewed HCC cases using DSA and triphasicMDCT Technique in our institution.

It has been recognized that the majority of HCCs are hypervascular. During the hepatic arterial phase (HAP), hypervascular lesions are greatly enhanced, and become iso- or hypodense in the portal venous phase (PVP), which is a sensitive and specific feature for diagnosing HCC. A tiphasic hepatic acquisition helical CT scanning technique has become a standard method for clinical diagnosis of HCC (Baron RL 1996; Oliver JH 3rd 1996; Paulson EK 1998; Mitsuzaki K 1996). Meanwhile, hepatic angiography is widely used as an imaging technique for HCC.It has been known that the imaging techniques for detection and staging of HCC take advantage of two basic events: 1) the disappearance of portal vessels, which are substituted with arterial vessels; and 2) a progressive disappearance of the reticuloendothelial system (RES). MDCT has a remarkably greater sensitivity in detecting small neoplastic nodules $(<20 \mathrm{~mm})$. The advantage of MDCT scan is mainly due to the possibility of imaging the liver in the arterial phase(Zheng XH 2005). 
In this study, all 30 patients had at least one overt HCC. We found that during MDCT, however, even with the most well timed IV bolus injection, the contrast medium is substantially diluted and scattered by the time it reaches the hepatic artery thus isolating a true arterial phase and a true portal venous phase may be difficult or even impossible. Since the triphasic helical CT does not have $100 \%$ sensitivity for detection of HCC, some lesion are liable to be missed especially the early HCC, as mentioned by Takayasu et al.(Yaqoob J 2004)which results in false-negative findings.

We examined our CT data on a lesion-by-lesion basis; we found that CT missed 10 of $34 \mathrm{HCC}$ lesions measuring $1.5 \mathrm{~cm}$ or less. Another tumor nodule, which measured $2.8 \mathrm{~cm}$, was not visible on CT. False negative results due to artifacts, partial-volume averaging, respiratory motion, and the rare iso-attenuating lesion at hepatic arterial and portal venous phases are additional factors that can lower the sensitivity of CT in the detection of HCCs (Teefey SA 2003).

CT findings are at times nonspecific and difficult to differentiate other hypervascular tumors from HCC.17 Therefore, MDCT has known limitations for the detection of liver lesions, especially small hypervascular lesions(Vallset al, 2004) evaluated the diagnostic accuracy of contrast-enhanced helical CT in 84 patients with liver cirrhosis before liver transplantation, using a contrast injection rate of $5 \mathrm{~mL} / \mathrm{s}$. Compared with the histopathologic results, the overall detection rate was $79 \%$; however, the detection rate of lesions with a diameter less than $2 \mathrm{~cm}$ was $61 \%$. Thismay offer an explanation for the higher detection rate of hypervascular masses on digital subtraction angiography compared with that on contrast-enhanced CT.

Conventional angiogram generates substantially more information than contrast enhanced CT triphasic.It delineates the exact location of the target tumor than perinterventional CT and MRI.

The Angiogram should, thus be able to provide delineation of tumorfeeding vessels and information about tumor location and extent, both of which could significantly influence the course of the intervention. Hepatic angiography has a dual advantage,duringreal time imaging we can generate a treatment plan which helps theradiologistto optimally block tumor feeding vessel.

In this study, we evaluated whether Conventional angiogram provides the same information regarding hepatic tumor spread in patients with hypervascular tumors as does state-of-the-art MDCT. Both methods were used to assess the extent of hepatic tumor involvement before TACE. Conventional angiogram may even increase the oncologic safety of the procedure because some false positive lesions seen on Conventional Angiogram might have been missed on MDCT.On the basis of the poor performance of CT for detecting small hypervascular lesions, some of the false-positive findings on C.A might actually have indicated true lesions that were not detected on MDCT (Peterson MS 2000; Iwazawa J 2009).

We examined our CT data on a lesion-by-lesion basis; we found that CT missed 10 of $34 \mathrm{HCC}$ lesions measuring $1.5 \mathrm{~cm}$ or less. Another tumor nodule, which measured $2.8 \mathrm{~cm}$, was not visible on CT.

Several limitations of our current study should be acknowledged. The most important is whether a lesion is the real target tumor or simply non tumor enhancement of DSA images is sometimes difficult to determine. Cirrhosis can cause inhomogeneous enhancement, resulting in enhanced pseudolesions, and organs such as the adrenal gland or gallbladder occasionally mimic true lesions. Furthermore we can not generate accurate treatment plan as MDCT can. A furtherlimitation of this study is poor contrast resolution and lack of a true standard of reference for all lesions(Capasso P 1996).

Transarterialchemoembolizations require high spatial resolution and real-time imaging to guide and monitor the intervention. As in almost all percutaneous endovascular procedures, this is best accomplished using fluoroscopy. However, a major disadvantage of fluoroscopy and angiography compared with cross-sectional imaging techniques such as CT or MRI is the possibility of missing softtissueinformation regarding the target organs (Capasso P 1996).

Consequently the true- sensitivity, specificity and accuracy of triphasic helical CT was also not possible. The results of our study show that the sensitivity of Hepatic angiograms is $75-80 \%$ in detectingnumber of lesions than those of CT triphasic. However this method being invasive is not commonly used in practice.

\section{REFERENCES}

- Baron RL, Oliver JH 3rd, Dodd GD 3rd, Nalesnik M, HolbertBL,Carr B.(1996) Hepatocellular carcinoma: evaluation with biphasic, contrast-enhanced, helical CT. Radiology; 199: 505511.

- Capasso P, Trotteur G, Flandroy P, DondelingerRF.(1996) A combined CT and angiography suite witha pivoting table. Radiology; 199:561-563

- Forner A, Llovet JM, Bruix J.(2012). Hepatocellular carcinoma. Lancet. 379:1245-55.

- Iwazawa J, Ohue S, Hashimoto N, Abe H, Hamuro M, Mitani T.(2010)AJR Am J Roentgenol. 195:882-7.

- Iwazawa J, Ohue S, Mitani T, Abe H, Hashimoto N, Hamuro M, et al. (2009Apr) Identifying feeding arteries during TACE of hepatic tumors: compa rison of $\mathrm{C}$-arm $\mathrm{CT}$ and digital subtractionangiography. AJR Am J Roentgenol. ;192(4):1057-63.

- Kausar S, Shafqat F, Shafi F, Khan AA.(1998). The association of hepatocellular carcinoma with hepatitis B and C viruses. Pak J Gastroenterol 12: 1-3.

- Llovet JM, Di Bisceglie AM, Bruix J, Kramer BS, Lencioni R, Zhu AX, Sherman M, Schwartz M, Lotze M, Talwalkar J, Gores GJ.(2008). Panel of Experts in HCC-Design Clinical Trials. Design and endpoints of clinical trials in hepatocellular carcinoma. J Natl Cancer Inst. 100:698-711.

- Meyer BC, Frericks BB, Voges M, Borchert M, Martus P, Justiz J, et al. (2008) Visualization of hypervascular liver lesions During TACE: compari son of angiographic C-arm CT and MDCT. AJR Am J Roentgenol. ;190:263-9.

- Mitsuzaki K, Yamashita Y, Ogata I, Nishiharu T, Urata J, Takahashi M.(1996) Multiple-phase helical CT of the liver for detecting small hepatomas in patients with liver cirrhosis: contrastinjection protocol and optimal timing. Am J Roentgenol; 167: 753757

- Oliver JH 3rd, Baron RL, Federle MP, and Jones BC, Sheng R.(1997)Hypervascular liver metastases: do unenhanced and hepatic arterial phase CT images affect tumor detection? Radiology; 205: 709-715

- Oliver JH 3rd, Baron RL, Federle MP, Rockette HE Jr.(1996). Detecting hepatocellular carcinoma: value of unenhanced or arterial phase CT imaging or both used in conjunction with conventional portal venous phase contrast-enhanced CT imaging. AmJRoentgenol; 167: 71-77.

- Oliver JH 3rd, Baron RL.(1996) Helical biphasic contrast-enhanced CT of the liver: technique, indications, interpretation, and pitfalls. Radiology; 201: 1-14

- Paulson EK, McDermott VG, Keogan MT, DeLong DM, Frederick MG, Nelson RC. (1998) Carcinoid metastases to the liver: role of triple-phase helical CT. Radiology; 206: 143-150

- Peterson MS, Baron RL, Marsh JW Jr, Oliver JH 3rd, Confer SR, Hunt LE.(2000)Pretransplantation surveillance for possible hepatocellular carcinoma in patients with cirrhosis: epidemiology and CTbased tumor detection rate in 430 cases with surgical pathologic correlation. Radiology; 217:743-749.

- Teefey SA, Hildeboldt CC, Dehdashti F, Siegel BA, Peters MG, Heiken JP, et al.(2003) Detection of primary hepatic malignancy in liver transplant candidates: prospective comparison of CT, MR imaging, US, and PET. Radiology. ;226:533-42. 
- Transarterial Catheter Chemoembolization. Available http://en.wikipedia.org/wiki/Transarterial_chemoembolization Accessed on June 2013.

- Valls C, Cos M, Figueras J, Andía E, Ramos E, Sánchez A, et al.(2004)Pretransplantation diagnosis and staging of hepatocellular carcinoma in patients with cirrhosis: value of dual-phase helical CT. AJR Am J Roentgenol.;182:1011-7.

- Wang Y, Shen Y.(2013).Unresectable hepatocellular carcinoma treated with transarterial chemoembolization: clinical data from a single teaching hospital.Int J ClinExp Med.;6:367-71.

- Xu, K. C., Niu, L. Z., Zhou, Q., Hu, Y. Z., Guo, D. H., Liu, Z. P., ... \&Zuo, J. S. (2009). Sequential use of transarterial
- chemoembolization and percutaneous cryosurgery for hepatocellular carcinoma. World journal of gastroenterology: WJG, 15(29), 3664.

- Yaqoob J, Bari V, Usman M U, Munir K, Mosharaf F, Akhtar W.(2004)The Evaluation of Hepatocellular Carcinoma with Biphasic Contrast enhanced Helical CT Scan J Pak Med Assoc. ;54:123-7.

- Zheng XH, Guan YS, Zhou XP, Huang J, Sun L, Li X, et al. (2005) Detection of hypervascular hepatocellular carcinoma: Comparison of multi-

detector CT with digital subtractionangiography and Lipiodol CT. World J Gastroenterol.;11:200-3. 